\title{
Synergistic interactions in mixed-species biofilms of pathogenic bacteria from the respiratory tract
}

\author{
Maryam Varposhti ${ }^{[1]}$, Fatemeh Entezari ${ }^{[1]}$ and Mohammad Mehdi Feizabadi ${ }^{[1]}$
}

[1]. Department of Microbiology, School of Medicine, Tehran University of Medical Sciences, Tehran, Iran.

\begin{abstract}
Introduction: Mixed-species biofilms are involved in a wide variety of infections. We studied the synergistic interactions during dual-species biofilm formation among isolates of Pseudomonas aeruginosa, Acinetobacter baumannii, and Stenotrophomonas maltophilia. Methods: Isolates were cultured as single-species and all possible combinations of dual-species biofilms. Results: The $61 \mathrm{~A}$. baumannii biofilms increased by 26-fold when cultured with $S$. maltophilia isolates; 62 A. baumannii biofilms increased by 20 -fold when cultured with $S$. maltophilia isolates; and 31 P. aeruginosa biofilms increased by 102 -fold when cultured with S. maltophilia 106. Conclusions: Synergy was observed between two isolates, including those that inherently lacked biofilm formation ability.
\end{abstract}

Keywords: Chronic infection. Mixed-species biofilm. Synergy.

Biofilms are groups of bacteria encased in a self-produced extracellular polymeric matrix ${ }^{1}$, and which consist of superficial microbial colonies that attach to solid surfaces. Biofilms cause a variety of persistent infections, including chronic middle ear infections, chronic sinusitis, chronic otitis, and lung infections in people with the inherited disease, cystic fibrosis (CF). The microcolonies that constitute the biofilm can be composed of singlespecies populations or multimember communities of bacteria, depending on the environmental parameters under which they are formed ${ }^{2}$. Different bacteria inside a biofilm structure are in close contact with each other; they display multiple phenotypes and have multiple genotypes, which arise by horizontal gene transfer. They also have quorum-sensing-specific effects on each other ${ }^{3}$.

Pseudomonas aeruginosa, Acinetobacter baumannii, and Stenotrophomonas maltophilia are among the most important causative agents in nosocomial respiratory diseases in Tehran hospitals. Treatment of these infections is difficult, as they show high levels of intrinsic or acquired resistance to different antimicrobial agents, markedly reducing the antibiotic options available for treatment $t^{4,5}$. Bacterial species isolated from cases with respiratory tract infections usually show strong resistance to antimicrobial agents; this could be related to the potential for biofilm formation in respiratory tract. According to Starner et al. ${ }^{6}$, bacterial biofilms are increasingly recognized as the cause of persistence and disease pathogenesis in respiratory infections, such as $\mathrm{CF}^{6}$.

Address to: Dr. Mohammad Mehdi Feizabadi. Department of Microbiology/ School of Medicine/Tehran University of Medical Sciences. Keshavarz Blvd, Porsina Ave, Tehran, Iran.

Phone: 9821 8895-5810; Fax: 9821 8895-5810

e-mail:mfeizabadi@sina.tums.ac.ir

Received 21 January 2014

Accepted 9 April 2014
We have previously reported the occurrence of hospitalacquired pneumonia with different organisms in Tehran hospitals. Polymicrobial infections have been observed in such patients ${ }^{4}$. The aim of this study was to evaluate the occurrence of synergistic interactions during dual-species biofilm formation by the bacteria isolated from respiratory tract of these patients.

Bacterial strains, isolated from patients who were hospitalized in Tehran University Hospitals (Tehran, Iran), were screened for their biofilm production potential. The screening process was performed in 96-well microtiter plates using the crystal violet method ${ }^{7,8}$. Six bacterial strains, including P. aeruginosa $(\mathrm{n}=2), A$. baumannii $(\mathrm{n}=2)$, and S. maltophilia $(\mathrm{n}=2)$ were selected for single- and dual-species biofilm formation. These organisms were isolated from patients with poly-microbial infections in the lower respiratory tract.

Dual-species biofilm formation was assessed on Foley catheter pieces ${ }^{9,10}$. Briefly, discs of catheter material (surface area: $0.5 \mathrm{~cm}^{2}$ ) were cut from catheters, with sterile cutters under aseptic conditions, and placed in 24-well Nunclon (Thermo Fisher Scientific, Waltham, MO) tissue culture plates. A standardized cell suspension $(80 \mu \mathrm{L})$ was applied to the surface of each disc, for dual species biofilms $40 \mu \mathrm{L}$ of each bacterial suspension were added and the discs were incubated for $1 \mathrm{~h}$ at $37^{\circ} \mathrm{C}$ (adhesion period). Non-adherent organisms were removed by gentle washing with $0.15 \mathrm{M}$ PBS $(5 \mathrm{~mL})$, and the discs were submerged in $1 \mathrm{~mL}$ of brain heart infusion broth (BHIB), then incubated for $24 \mathrm{~h}$ at $37^{\circ} \mathrm{C}$ to allow biofilm formation. In control experiments, discs lacking cells were incubated in medium containing $1 \mathrm{~mL}$ BHIB. All biofilm and control assays were carried out three times, in triplicate. Quantitation of biofilm growth was measured using a tetrazolium reduction assay with tetrazolium salt 3-[4,5-dimethylthiazol-2yl]-2,5-diphenyltetrazolium bromide (methylthiazol tetrazolium [MTT]; Sigma-Aldrich; St Louis, MO) ${ }^{11}$. 
After biofilm formation, $1 \mathrm{~mL}$ MTT solution $(0.3 \%$ in phosphate-buffered saline) was added to each well and subsequently incubated for $5 \mathrm{~h}$ at $37^{\circ} \mathrm{C}$. MTT was then removed, and the wells were washed three times with $0.15 \mathrm{M}$ PBS $(2 \mathrm{~mL})$ to remove all traces of MTT. Dimethyl sulfoxide $(1 \mathrm{~mL})$ was then added to solubilize the MTT formazan product. Then, dimethyl sulfoxide was transferred to 96-well microtiter plates and MTT formazan formation was measured at $550 \mathrm{~nm}$ using an enzyme-linked immunosorbent assay (ELISA) reader (Anthos, Australia). Control wells containing medium plus MTT were used to determine background formazan values. All assays were carried out three times in triplicate.

Each of these six isolates was grown as single-species biofilms as well as in all possible combinations of two different species. Synergistic interactions were observed in the majority of combinations. With 18 combinations, biofilm formation by two species was significantly greater than biofilm production by any of the single species. According to Table 1, more than $100 \%$ increase in biofilm formation was observed in 14 out of 24 combinations. These included combination of isolates with low activity in making biofilm (Table 2). The results show that the biomass of a multi-species biofilm is not necessarily the sums of the biomass of each single species. Although P. aeruginosa 31 and $A$. baumannii 62 did not strongly produce biofilm (as clearly seen Figure 1), a considerable amount of biofilm (20-fold increase) was formed when these strains were grown in the presence of $S$. maltophilia (Figure 1). The strongest synergism was observed between $P$. aeruginosa 31 and $S$. maltophilia 106: the biofilm biomass of P31 was increased by more than 100-fold. The total number of cells remained constant during inoculation for both single- and dual-species biofilm and the optical density values indicated the biofilm biomass ${ }^{8}$.

Polymicrobial infections constitute a high percentage of nosocomial respiratory infections and cause serious problems in therapeutic procedures. In the present study, using 12 different combinations, the biofilm formation when two species were present was significantly greater than biofilm production by any of the species alone. Synergy was observed even between those who were not able to produce strong biofilm on their own. Therefore, the infections caused by mixed bacterial populations can increase the disease intensity via strong biofilm production. For instance, we demonstrated that $S$. maltophilia is able to induce biofilm production in $P$. aeruginosa 31 , which was not able to produce biofilms by itself.

Since the total number of cells was kept constant during inoculation for both single- and dual-species biofilms, it became obvious that diversification is more effective than the number of cells involved in the primary stages of biofilm formation. Thus, in a niche with a specified number of cells, the presence of different species of bacteria can result in a greater quantity of biofilm, as has been reported previously ${ }^{12,13}$.

Synergy in biofilm formation by bacteria involved in a mixed-species structure could be related to the co-aggregation process. Biofilm formation of some non-co-aggregating bacteria can be promoted by other strains in an environment with bacterial diversity. In an investigation of dental plaques, it was
TABLE 1 - Percentage of increase in the amount of biofilm formation of individual bacterial strains when combined with the other bacterial strains.

\begin{tabular}{lcccccc}
\hline & P30 & P31 & A61 & A62 & S106 & S107 \\
\hline P30 & - & - & 4 & 222.3 & 15.15 & 6.9 \\
P31 & - & - & 13.79 & 333.3 & 212.1 & 69.76 \\
A61 & 0 & 3,200 & - & - & 233.2 & 155.81 \\
A62 & 115 & 1,200 & - & - & 81.81 & 39.02 \\
S106 & 18.75 & 10,200 & 279.3 & 2,000 & - & - \\
S107 & 43.75 & 7,200 & 279.3 & 2,666 & - & - \\
\hline
\end{tabular}

P30: Pseudomonas aeruginosa 30; P31: Pseudomonas aeruginosa 31; A61: Acinetobacter baumannii 61; A62: Acinetobacter baumannii 62; S106: Stenotrophomonas maltophilia 106; S107: Stenotrophomonas maltophilia 107 . We did not combine the same bacterial strains; thus, some places in the table show no data.

TABLE 2 - MTT optical density values of single- and dual-species biofilms.

\begin{tabular}{lc}
\hline Bacterial species & Biofilm amount $\left(\mathrm{OD}_{550} \mathrm{~nm}\right)$ \\
\hline $\mathrm{P} 30$ & 0.0032 \\
$\mathrm{P} 31$ & 0.0001 \\
$\mathrm{~A} 61$ & 0.0029 \\
$\mathrm{~A} 62$ & 0.0003 \\
$\mathrm{~S} 106$ & 0.0033 \\
$\mathrm{~S} 107$ & 0.0043 \\
$\mathrm{P} 30+\mathrm{A} 61$ & 0.0029 \\
$\mathrm{P} 30+\mathrm{A} 62$ & 0.0069 \\
$\mathrm{P} 31+\mathrm{A} 61$ & 0.0033 \\
$\mathrm{P} 31+\mathrm{A} 62$ & 0.0013 \\
$\mathrm{P} 30+\mathrm{S} 106$ & 0.0038 \\
$\mathrm{P} 30+\mathrm{S} 107$ & 0.0046 \\
$\mathrm{P} 31+\mathrm{S} 106$ & 0.0103 \\
$\mathrm{P} 31+\mathrm{S} 107$ & 0.0073 \\
$\mathrm{~A} 61+\mathrm{S} 107$ & 0.0109 \\
$\mathrm{~A} 61+\mathrm{S} 106$ & 0.0109 \\
$\mathrm{~A} 62+\mathrm{S} 106$ & 0.0063 \\
$\mathrm{~A} 62+\mathrm{S} 107$ & 0.0083 \\
\hline $\mathrm{P} 30:$ Pseu & \\
\hline
\end{tabular}

P30: Pseudomonas aeruginosa 30; P31: Pseudomonas aeruginosa 31; A61: Acinetobacter baumannii 61; A62: Acinetobacter baumannii 62; S106: Stenotrophomonas maltophilia 106; S107: Stenotrophomonas maltophilia 107. MTT: methylthiazol tetrazolium. OD $_{550}$ : optical density at $550 \mathrm{~nm}$. 


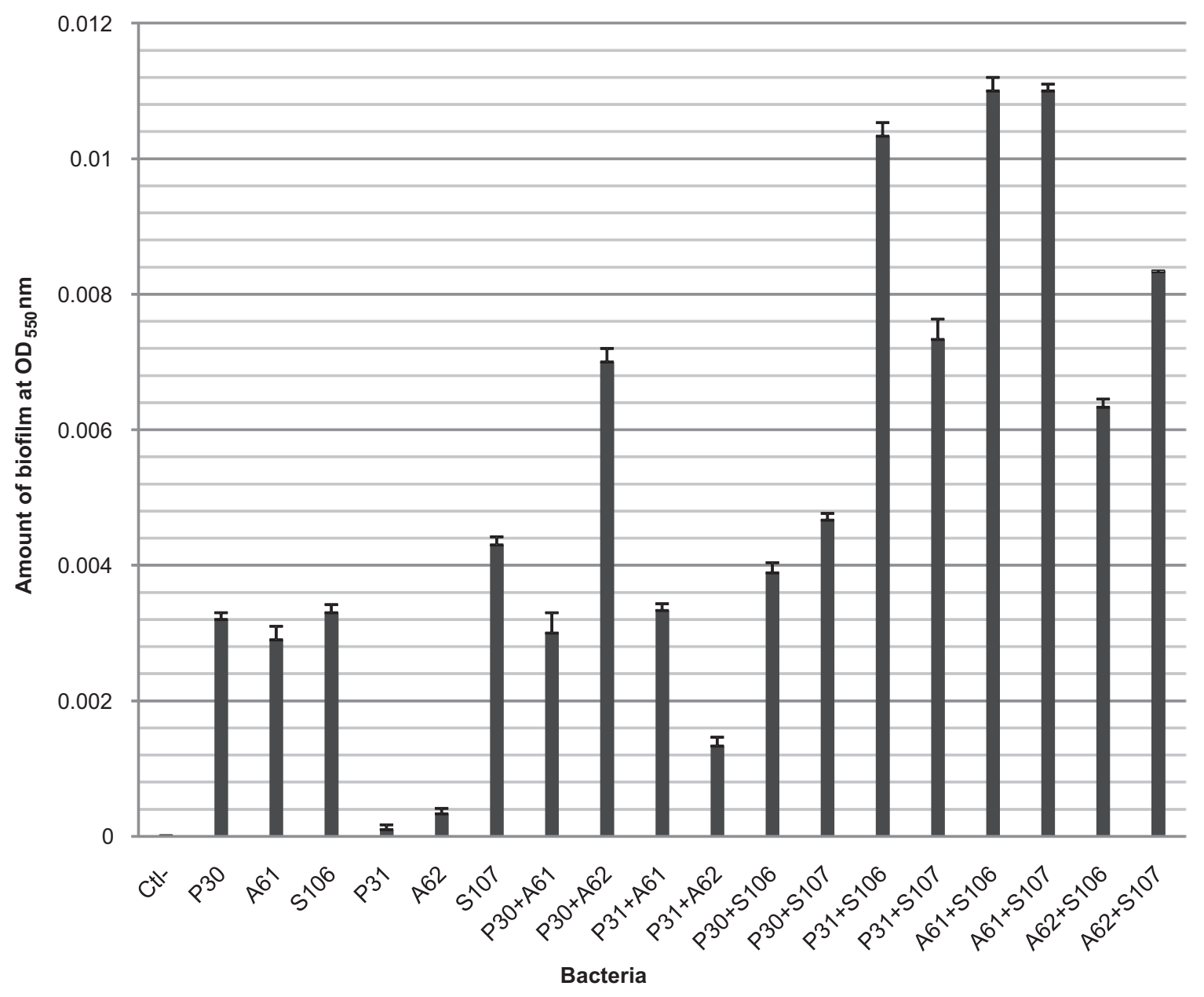

FIGURE 1 - Biomass of single- and dual-species biofilms. The mean \pm standard deviation for nine replicates is illustrated. OD $_{550}$ : optical density at 550nm. P30: Pseudomonas aeruginosa 30; P31: Pseudomonas aeruginosa 31; A61: Acinetobacter baumannii 61; A62: Acinetobacter baumannii 62; S106: Stenotrophomonas maltophilia 106; S107: Stenotrophomonas maltophilia 107.

observed that Actinomyces species could induce biofilm formation of other non-co-aggregating bacteria; thus, the presence of Actinomyces is critical in dental plaque formation ${ }^{14}$. One probable explanation for the marked increase in biofilm formation by P31 and A62 isolates may be the enhanced attachment of these cells that occurs after the attachment of S. maltophilia to the surface.

Expression of some features only in mixed-species biofilms is another remarkable characteristic of bacterial cells in such structures. In an investigation on chronic rhinosinusitis, it was observed that Haemophilus influenzae expresses its virulence factor type IV pili (pilA) only in mixed-species biofilms. In the same niche, H. influenzae and Streptococcus pneumoniae supported each other in adhering to the host epithelia and enhanced the eventual establishment of a recalcitrant polymicrobial community ${ }^{15}$.

In this study, bacteria from different genera were used together and inter-species communication between the strains, including the secreted factors (quorum-sensing molecules, secondary metabolites, carbohydrates, and proteins), which influence gene expression, metabolic co-operation and competition, physical contact, and the production of antimicrobial exoproducts, may lead to enhanced biofilm formation. The exact mechanism of synergism between these three bacteria is not known at present and one or more of the interactions mentioned above may have occurred during this experiment.

These results may suggest that studying the interactions between pathogens in respiratory tract infections could facilitate the more suitable and effective in future. A future challenge in biofilm research will be the dissection of these interactions.

\section{CONFLICT OF INTEREST}

The authors declare that there is no conflict of interest.

\section{REFERENCES}

1. Greenberg EP. Bacterial communication and group behavior. J Clin Invest 2003; 112:1288-1290.

2. Costerton JW, Stewart PS, Greenberg EP. Bacterial biofilms: a common cause of persistent infections. Science 1999; 284:1318-1322. 
3. Ehrlich GD, Hu FZ, Shen K, Stoodley P, Post JC. Bacterial plurality as a general mechanism driving persistence in chronic infections. Clin Orthop Relat Res 2005; 20-24.

4. Feizabadi MM, Majnooni A, Nomanpour B, Fatolahzadeh B, Raji N, Delfani S, et al. Direct detection of Pseudomonas aeruginosa from patients with healthcare associated pneumonia by real time PCR. Infect Genet Evol 2010; 10:1247-1251.

5. Nicodemo AC, Paez JI. Antimicrobial therapy for Stenotrophomonas maltophilia infections. Eur J Clin Microbiol Infect Dis 2007; 26:229-237.

6. Starner TD, Zhang N, Kim G, Apicella MA, McCray Jr PB. Haemophilus influenzae forms biofilms on airway epithelia: implications in cystic fibrosis. Am J Respir Crit Care Med 2006; 174:213-220.

7. Yarwood JM, Bartels DJ, Volper EM, Greenberg EP. Quorum sensing in Staphylococcus aureus biofilms.J Bacteriol 2004; 186:1838-1850.

8. Quave CL, Plano LR, Pantuso T, Bennett BC. Effects of extracts from Italian medicinal plants on planktonic growth, biofilm formation and adherence of methicillin-resistant Staphylococcus aureus. J Ethnopharmacol 2008; 118:418-428.

9. Adam B, Baillie GS, Douglas LJ. Mixed species biofilms of Candida albicans and Staphylococcus epidermidis. J Med Microbiol 2002; 51:344-349.
10. Hawser SP, Douglas LJ. Biofilm formation by Candida species on the surface of catheter materials in vitro. Infect Immun 1994; 62:915-921.

11. Walencka E, Sadowska B, Rozalska S, Hryniewicz W, Rozalska B. Lysostaphin as a potential therapeutic agent for staphylococcal biofilm eradication.Pol J Microbiol 2005; 54:191-200.

12. Lopes SP, Ceri H, Azevedo NF, Pereira MO. Antibiotic resistance of mixed biofilms in cystic fibrosis: impact of emerging microorganisms on treatment of infection. Int J Antimicrob Agents 2012; 40:260-263.

13. Burmolle M, Webb JS, Rao D, Hansen LH, Sorensen SJ, Kjelleberg S. Enhanced biofilm formation and increased resistance to antimicrobial agents and bacterial invasion are caused by synergistic interactions in multispecies biofilms. Appl Environ Microbiol 2006; 72:3916-3923.

14. Filoche SK, Anderson SA, Sissons CH. Biofilm growth of Lactobacillus species is promoted by Actinomyces species and Streptococcus mutans. Oral Microbiol Immunol 2004; 19:322-326.

15. Cope EK, Goldstein-Daruech N, Kofonow JM, Christensen L, McDermott B, Monroy F, et al. Regulation of virulence gene expression resulting from Streptococcus pneumoniae and nontypeable Haemophilus influenzae interactions in chronic disease. PLoS One 2011; 6: e28523. 